\title{
Smart Homes Design for People with Dementia
}

\author{
Mohsen Amiribesheli \\ Department of Computing \& Informatics \\ Faculty of Science and Technology \\ Bournemouth University, UK \\ Email: mamiribesheli@bournemouth.ac.uk
}

\author{
Abdelhamid Bouchachia \\ Department of Computing \& Informatics \\ Faculty of Science and Technology \\ Bournemouth University, UK \\ Email: abouchachia@bournemouth.ac.uk
}

\begin{abstract}
In this paper, we present a user-centred approach for designing and developing smart homes for people with dementia. In contrast to most of the existing literature related to dementia, the present approach aims at tailoring the system to the specific needs of dementia using a scenario-based methodology. Scenarios are based on typical dementia symptoms which are collected from research literatures and validated by dementia caregivers. They portray the common behaviour of people with dementia. Because they explain real-world situations, scenarios are meant to generalise the requirements of smart homes for people with dementia. Hence, a top-down approach is followed to summarise the content of the scenarios into the essential requirements for smart home frameworks dedicated to monitoring people with dementia.
\end{abstract}

\section{INTRODUCTION}

Expectations show that one in five people will be older than 65 by 2030 [1] and statistics reveal that about $3 \%$ of people aged 65 to $74,19 \%$ of people aged 74 to 84 and nearly half of people more than 84 will have dementia [2]. Most of them, however, strongly prefer to stay in their own homes and communities, a phenomenon known as age-in-place. It has been observed that age-in-place can reduce the speed of dementia progress, improve people's quality of life [3] and enable them to be surrounded by their families. However, informal care at home can be excessively expensive and in some cases it is not possible at all [4]. Taking stock of the difficulties associated with informal care, the use of assisted living technologies such as smart homes (SHs) can substantially help a person with dementia (PWD) to live independently. A smart home environment is usually equipped with a collection of inter-related software and hardware components to monitor the living space by capturing the behaviours of the resident and understanding his activities. By doing so, the system can inform about risky situations and take actions on behalf of the resident to his satisfaction [5]. An SH can be employed for different types of care: informal by family members, formal by hospital staffs and social by caregivers.

Dementia is defined as a family of chronic diseases that cause permanent and gradual cognitive decline. It comes with one or more of the following symptoms [6]:

- decrease in the ability of speaking or comprehending spoken or written language,

- difficulties to distinguish or identify objects,

- difficulties in performing motor activities,

- difficulties for reasoning abstractly, making valid judgements and planning for complicated tasks.
The most common type of dementia is the Alzheimer's Disease (AD). The disease is more likely to occur in old age and as the elderly population grows, the proportion of people with $\mathrm{AD}$ increases. Although some medications can slow the progress of the disease, currently there is no way to stop its development altogether or reverse its impacts on the brain cells. The pace of the dementia progress is specific to the individuals and even the symptoms may not be the same for them. However, a study [7] allowed to categorise the dementia progress into 7 stages: no cognitive decline, very mild cognitive decline, mild cognitive decline (Mild Cognitive Impairment MCI), moderate cognitive decline (Mild Dementia), moderately severe cognitive decline, severe cognitive decline, and very severe cognitive decline.

Ideally an SH system should be adapted to the dementia category of the resident. Towards this aim, Orpwood et al. [8] analysed SH requirements of PWDs using a qualitative research methodology and involving interviews and focus groups. The outcome of the analysis can be summarised as follows: a) PWDs cannot learn to use new devices in their home, b) new equipment needs to look like a previously seen one, c) PWDs need to maintain a sense of control over their environment, d) new technologies should be first evaluated by caregivers, and e) it is important to support caregivers by providing prompts and reminders. These requirements served to design some SH applications such as a reminder, a cooking monitor and a lighting system.

In [9], the authors conducted a series of qualitative research studies about people with mild to moderate dementia and their caregivers in order to collect their requirements in the home environment. The authors found out that PWDs need support for dressing, taking medicine, maintaining personal hygiene, preparing food and socialising. Van Hoof et al. [10] investigated the effectiveness of SHs for age-in-place using interviews and observation. In this study an $\mathrm{SH}$ prototype was developed and used to analyse a group of old people. The prototype is equipped with various functionalities like movement monitoring, fire detection, wandering detection and fall detection. It was found that PWDs appreciate safety and security, especially fall detection. Mihailidis et al. [11] studied the application of SHs for the purpose of assisting people with moderate dementia in the accomplishment of daily living activities. The authors evaluated an audio-visual system dedicated to the task of hand washing in a bathroom. Cohene et al. [12] employed a set of user-centred design (UCD) tools to develop an interactive digital multimedia life stories system for PWDs. They suggested interacting with the system could enhance the PWD's well-being and improve their memory capabilities. While designing the system, they altered the conventional UCD 
tools for eliciting a PWD's requirements. In the research, the effects of simplicity, stimulation, and active participation on the quality of the system were discussed.

In addition to the studies on the process of designing smart applications for the PWD monitoring, there exists a body of literature on SH architectures. In the study [13], an architecture for healthcare monitoring in smart hospitals is suggested. It adapts an SOA-based (service-oriented architecture) design to support applications that employ a variety of sensors and actuators. The architecture proposes a loosely-coupled system including UPnP and semantic web. Lin et. al. [14] produced an $\mathrm{SH}$ architecture using SOA approach. The proposed system applies OSGi in a way that its components can communicate with all other primary non-OSGi platforms through different types of network. Another study [15] introduced and developed an architecture for SHs using SOA and web-services. The authors evaluated the system by adopting a series of qualitative research methods. Results illustrated that users had a positive attitude toward the SHs employing the proposed architecture.

Considering the diversity and peculiarities of SH stakeholders, having extensive attention to their needs, wants and limitations is essential. For instance, the learning curve of a person with dementia gets altered by the disease; thus it takes a much longer time for PWDs to learn a simple interaction, and it is much easier for them to forget it. For taking these into account, a design process should be employed that guarantees the system entirely fits the users' requirements, and the users are not forced into adapting themselves to the system by learning how to perform new interactions. This process of designing an SH involving the targeted people is known as user-centred. There exists a number of tools for performing a user-centred design study and tools such as personas and scenarios [16]. Personas are fictional characters based on real stakeholders, and scenarios are the written details of a persona's interaction with the system and the environment. These tools are employed for iterative analysis of the system usability by stakeholders and designers and to develop the system after the analysis.

In this study, the main personas characterise the PWD. These personas are based on the typical dementia symptoms in various stages of the disease and they are obtained from the research literatures. The scenarios are based on the persona's actions and the system reactions to them. Finally, using a topdown approach on the scenarios, the key requirements of an $\mathrm{SH}$ framework for PWDs are elicited.

The rest of the paper is structured as follows. Section II portrays the user-centred analysis tools which are employed in this research. Section III discusses our preliminary findings and initial results. Section IV concludes our work and presents the work to be undertaken in the future.

\section{USER-CENTRED DESIGN}

The user-centred design is an umbrella term that comprises a set of design tools and methods to involve the end-user of a system to specify their requirements regarding that system. Therefore, stakeholders should be participating in all stages of the design of the SH including analysis, design and evaluation. This paper is concerned with the analysis and design phases. Bearing in mind the peculiarities of dementia disease symptoms (e.g., memory restraints, mobility difficulties), it is considerably challenging to involve PWDs in the analysis and design steps. Moreover, the collected information from the PWD might not be accurate. Nevertheless, after developing the SH system, the PWD should participate in its evaluation and provide routes for quality improvement [8]. On the contrast, the other users of SH, especially informal and formal caregivers are usually well informed about the PWD's needs and preferences. The following describes two user-centred design tools of personas and scenario-based use cases.

Scenarios are defined as a tangible description of activities that users engage in when performing specific tasks, a description that is sufficiently detailed so that design implications can be understood and reasoned about [17]. During the scenarios development, it should be taken into consideration that it is unlikely for PWDs to encounter all of the disease symptoms. Hence, scenario identification solely based on qualitative research studies (e.g., interviews and focus groups) could be restricted by the small number of participants and their particular experiences [18]. In this study, the process of collecting scenarios consists of the following steps: first, all dementia symptoms are collected from the literature. Then, the scenarios are developed based on symptoms of each dementia stage using different personas. Finally, the scenarios are validated by formal and social caregivers.

This study used the following validation approach. The scenarios were validated by four social caregivers and two dementia specialists. An interview session was held for each stakeholder. While reading the scenarios, they were asked to modify the scenarios details based on their individual experiences. As the results of the interviews, the scenarios were enriched with details, and in few cases new scenarios were developed. For instance, "personal grooming" scenario was not on the initial list of scenarios and it was inserted after a number of suggestions from social caregivers were obtained. Ultimately, 13 scenarios were developed and validated. However, only three of them (without any particular priorities) are stated here due to space limitation.

The concept of intervention levels in the scenarios supports the PWD's independence and guarantees the PWD's sense of control [19]. In this research, five levels of intervention are considered: invites awareness, suggests, prompts, urges, and performs. These levels are modelled from the caregivers' supporting approaches. Usually the caregivers are asked to have the least interference with the PWD's life and ideally SH systems should follow similar approaches.

1) Repetitive speech: Robert is 69 years old. He has MCI and he lives in his home with his family. He repeats his questions and statements inattentively. His doctor stated that the progress of dementia inevitably increases the number of speech repetitions.

Requirements associated: The system invites awareness. The system detects the event by analysing the collected data from sensors (e.g., microphone) [20]. There is a direct correlation between the frequency of repeating and the progress of the disease. The frequency outlines Robert's disease stage, particularly showing the transition from MCI to the early stages of the $\mathrm{AD}$. 
2) Dehydration: Dorothy is a 72-year-old woman with mild dementia. She lives in her home with her family. She often ignores drinking necessary daily amount of water and this causes her dehydration. The dehydration aggravates her memory symptoms [21]. Reminding her to drink the required amount of water is one of her caregivers' (children) responsibilities.

Requirements associated: The system invites awareness and suggests. The system suggests her to drink water and monitors the action via sensors (e.g., contact switch sensors, RFID) to make sure that she actually drinks the water.

3) Night-time wandering: Ruth is an 84-year-old woman with late-stage dementia and lives in a care-home. She often faces night-time wandering. She wakes up during the night and wanders inside or outside the house. Obviously, without an immediate action from a caregiver, her night-time wandering is a threat to her health.

Requirements associated: The system prompts, urges and performs. The system notices the behaviour through interpreting sensor readings (e.g., passive infra-red, GPS) and prompts her to return to bed or to go to the toilet. The prompt is employed with a familiar person's voice (e.g., her daughter), and it is delivered through familiar devices such as radio or TV [22]. If she keeps wandering and tries to leave the house, system triggers an alarm to a caregiver. If the caregivers do not respond or they are far away from the home, the system can lock the exit doors and call emergency contacts.

TABLE I. THE USE CASE TABLE FOR THE REPETITIVE SPEECH SCENARIO

\begin{tabular}{|l|l|}
\hline $\begin{array}{l}\text { Stakeholder's } \\
\text { Actions }\end{array}$ & World Reactions \\
\hline Robert talks. & $\begin{array}{l}\text { (1) Microphone records the voice and transmits it to } \\
\text { the processing unit. (2) Using the profiles, the system } \\
\text { detects the Robert's voice and recognise the statements }\end{array}$ \\
\hline $\begin{array}{l}\text { Robert repeats him- } \\
\text { self. }\end{array}$ & $\begin{array}{l}\text { (1) Microphone records the voice and transmits it to } \\
\text { the processing unit. (2) Using the profiles, the system } \\
\text { detects the Robert's voice and recognise the statements. } \\
\text { (3) The system recognises the repetition. (4) The sys- } \\
\text { tem counts the repetition and log it. }\end{array}$ \\
\hline $\begin{array}{l}\text { Dementia specialist } \\
\text { requests for the } \\
\text { repatriation logs. }\end{array}$ & $\begin{array}{l}\text { (1) The system produces a visual presentation of the } \\
\text { number of repetitions. }\end{array}$ \\
\hline
\end{tabular}

TABLE II. THE USE CASE TABLE FOR THE DEHYDRATION SCENARIO

\begin{tabular}{|l|l|}
\hline $\begin{array}{l}\text { Stakeholder's } \\
\text { Actions }\end{array}$ & World Reactions \\
\hline $\begin{array}{l}\text { Formal caregiver } \\
\text { sets the number of } \\
\text { drinking time per } \\
\text { day. }\end{array}$ & $\begin{array}{l}\text { (1) The system receives the number of times and saves } \\
\text { it to the database. }\end{array}$ \\
\hline $\begin{array}{l}\text { Dorothy drinks wa- } \\
\text { ter. }\end{array}$ & $\begin{array}{l}\text { (1) The sensors detect movements. (2) The system } \\
\text { recognises the activity of drinking water using the } \\
\text { activity recognition component. (3) The system detects } \\
\text { Dorothy is drinking water using the profiles manage- } \\
\text { ment component. (4) The system logs the number of } \\
\text { time she drinks. }\end{array}$ \\
\hline $\begin{array}{l}\text { Dorothy forgets } \\
\text { to drink enough } \\
\text { amount of water. }\end{array}$ & $\begin{array}{l}\text { (1) The system employs familiar devices to ask Dorothy } \\
\text { to drink sufficient amount of water. (2) If she kept } \\
\text { ignoring the system, it sends an alarm to the caregiver. }\end{array}$ \\
\hline $\begin{array}{l}\text { Dementia specialist } \\
\text { requests for the } \\
\text { repatriation logs. }\end{array}$ & $\begin{array}{l}\text { (1) The system produces a visual presentation of the } \\
\text { number of repetitions. }\end{array}$ \\
\hline \multicolumn{2}{|l}{} \\
\hline
\end{tabular}

A use case is a list of users' actions and the corresponding world reactions (See Tables I and II). It is developed to elicit the requirements from the scenarios. It displays all the interactions between the users, the environment and the system during the scenario. The interactions are based on the causeeffect concept. A use case table is produced by analysing each scenario.

As an example, Table I presents the actions of Robert, who is suffering from MCI, and the desired reactions of system. The reactions help determine the software components or functionalities the system should consist of. For instance, it can be clearly seen that a processing unit, a profile manager, and a context (voice) recognition are needed for the first scenario.

To accumulate the essential design considerations for the framework, all the non-functional requirements (e.g., scalability, interoperability) and all the functional requirements of the system collected from the use-cases should be analysed. The non-functional requirements play an important role in the $\mathrm{SH}$ system quality. For instance, considering the significance of health monitoring, any failure of the system can be exceedingly costly and the consequences can be irreversible. The system should be stable and reliable to avoid any damage to PWDs.

In the following section, the preliminary findings from the analysis of the scenarios and consideration of the nonfunctional requirements are summarised.

\section{PRELIMINARY FINDINGS}

As it has been mentioned earlier, there exists a number of $\mathrm{SH}$ architectures that are produced for a variety of applications such as healthcare monitoring and energy saving. Our proposed approach for developing an $\mathrm{SH}$ architecture is unique as it applies user-centred design tools in early stages, particularly in the design phase. Moreover, contrary to the majority of existing general-purpose architectures, our design approach only focuses on the requirements of PWDs. The outcomes of the process are the requirements of designing a framework for an SH for PWDs. In the current phase of the research, the scenarios are not studied for the purpose of developing specific applications to address scenarios requirements. They are considered to obtain the essential requirements for building a structure that can host applications which address various scenarios including the mentioned ones. Three principal requirements of modularity, distribution and invisibility were initially identified by studying the 13 scenarios. They distinguish the framework from other types of SH frameworks. Although considering these requirements improve the quality of users' experience for all types of SHs, not considering these requirements makes the developed SH impractical for PWDs.

Modularity: Systems with a modular architecture incorporate a group of components (modules) to perform. Commonly, these architectures enable users to add or remove the components needless of making significant changes in the current configurations of the system. As it was explained earlier, dementia has a progressive nature, and each person with dementia has unique care demands. Furthermore, the variety of required components in the use-case tables demonstrate the necessity of having a modular design. Hence, the system should be implemented adopting a modular approach to perform in a contextaware manner and to address the stakeholders' preferences. A very prominent modular approach that can satisfy these needs is the Service-Oriented Architecture (SOA). The SOA is an abstract design pattern for developing modular systems. It has a 
set of principles and methodologies for designing interoperable services, service requesters and service providers.

Distribution: SOA systems often utilise the client-server model to operate. In the model, all the $\mathrm{SH}$ processes run on a computing unit (Gateway). Putting all the processing loads on a machine produces issues for the $\mathrm{SH}$, particularly in areas such as reliability and adjustability. When the goal is to monitor a PWD (i.e., scenario of night-time wandering), reliability of the system is crucially important, as the failure of monitoring system can be immensely harmful. In the clientserver design, the gateway failure causes all the SH services to be unavailable. Distributed design methods prevent these problems to occur through allowing the processing load to be divided into different components of a system. The Mobile Agent is a distributed design approach that employs a blend of computer software and data communication techniques to enable software components (agents) to migrate between the host computers in a network. It allows the agents to accomplish their tasks regardless of the host that they are running on.

Invisibility: Putting unfamiliar devices (e.g., sensors, interfaces) in a PWD's living environment can cause him anxiety. To prevent the situation, all the physical components of an SH system must be as invisible (transparent) as possible. The system should stay invisible even during interventions in the PWD's life. For instance, the communication between the system and the PWD should occur employing familiar devices such as television and radio in scenarios such as night-time wandering and dehydration.

\section{CONCLUSION AND FUTURE WORK}

The paper presents a scenario-based approach for eliciting requirements of an SH for PWDs; it also elicits the essential requirements of an architecture based on the outcomes of a scenario-based user-centred design approach. The scenarios are developed based on existing literature and in collaboration with the caregivers through iterative validations. During scenario development, the system emulates the caregivers in matters such as the intervention level. The scenarios are transformed to use cases.These use cases, along with the involuntary requirements of PWDs, were used to elicit the essential requirements of an SH for PWDs.

This paper focused on the analysis and the design phases. A crucial step during the user-centred design process of an SH system for PWDs is to identify which stakeholders should be involved in the validation process. For the analysis phase, the formal and informal caregivers were involved. During this phase, the focus was on finding the details of dementia symptoms and refining the scenarios.

Future work will be concerned with the low-level design, the implementation and the evaluation of the SH system.

\section{REFERENCES}

[1] S. Wild, G. Roglic, A. Green, R. Sicree, and H. King, "Global prevalence of diabetes estimates for the year 2000 and projections for 2030," Diabetes care, vol. 27, no. 5, pp. 1047-1053, 2004.

[2] D. A. Umphred, R. T. Lazaro, M. Roller, and G. Burton, Neurological rehabilitation. Elsevier Health Sciences, 2013.

[3] M. P. Cutchin, "The process of mediated aging-in-place: A theoretically and empirically based model," Social Science and Medicine, vol. 57, no. 6, pp. 1077-1090, 2003.
[4] A. Wimo, B. Winblad, and L. Jönsson, "The worldwide societal costs of dementia: Estimates for 2009" Alzheimer's \& Dementia, vol. 6, no. 2 , pp. 98-103, 2010

[5] M. Amiribesheli, A. Benmansour, and A. Bouchachia, "A review of smart homes in healthcare," Journal of Ambient Intelligence and Humanized Computing, pp. 1-23, 2015.

[6] W. Thies and L. Bleiler, "2013 alzheimer's disease facts and figures." Alzheimer's \& dementia: the journal of the Alzheimer's Association, vol. 9, no. 2, pp. 208-245, 2013.

[7] B. Reisberg, S. H. Ferris, M. J. de Leon, and T. Crook, "The global deterioration scale for assessment of primary degenerative dementia." The American journal of psychiatry, 1982.

[8] R. Orpwood, C. Gibbs, T. Adlam, R. Faulkner, and D. Meegahawatte, "The design of smart homes for people with dementiauser-interface aspects," Universal Access in the information society, vol. 4, no. 2, pp. 156-164, 2005.

[9] J. P. Wherton and A. F. Monk, "Technological opportunities for supporting people with dementia who are living at home," International Journal of Human-Computer Studies, vol. 66, no. 8, pp. 571-586, 2008.

[10] J. Van Hoof, H. Kort, P. Rutten, and M. Duijnstee, "Ageing-in-place with the use of ambient intelligence technology: Perspectives of older users," International journal of medical informatics, vol. 80, no. 5, pp. 310-331, 2011.

[11] A. Mihailidis, J. N. Boger, T. Craig, and J. Hoey, "The coach prompting system to assist older adults with dementia through handwashing: An efficacy study," BMC geriatrics, vol. 8, no. 1, p. 28, 2008.

[12] T. Cohene, R. Baecker, E. Marziali, and S. Mindy, "Memories of a life: a design case study for alzheimers disease," Universal Usability, John Wiley \& Sons, pp. 357-387, 2007.

[13] V. Foo Siang Fook, J. Maniyeri, A. A. P. Wai, P. V. Thang, and J. Biswas, "Service oriented architecture for patient monitoring application," in Industrial Informatics, 2006 IEEE International Conference on. IEEE, 2006, pp. 160-165.

14] R.-T. Lin, C.-S. Hsu, T. Y. Chun, and S.-T. Cheng, "Osgi-based smart home architecture for heterogeneous network," in Sensing Technology 2008. ICST 2008. 3rd International Conference on. IEEE, 2008, pp. 527-532.

[15] E. Kaldeli, E. U. Warriach, A. Lazovik, and M. Aiello, "Coordinating the web of services for a smart home," ACM Transactions on the Web (TWEB), vol. 7, no. 2, p. 10, 2013.

[16] M. Aoyama, "Persona-and-scenario based requirements engineering for software embedded in digital consumer products" in Requirements Engineering, 2005. Proceedings. 13th IEEE International Conference on. IEEE, 2005, pp. 85-94.

[17] J. Van Helvert and C. Fowler, "Scenario-based user needs analysis," chimera working paper, vol. 2, 2003.

[18] K. Hawkey, K. M. Inkpen, K. Rockwood, M. McAllister, and J. Slonim, "Requirements gathering with alzheimer's patients and caregivers," in Proceedings of the 7th International ACM SIGACCESS Conference on Computers and Accessibility, ser. Assets '05. New York, NY, USA: ACM, 2005, pp. 142-149.

[19] C. A. Thompson, K. Spilsbury, J. Hall, Y. Birks, C. Barnes, and J. Adamson, "Systematic review of information and support interventions for caregivers of people with dementia," BMC geriatrics, vol. 7, no. 1 , p. 18,2007

[20] V. Nemes, D. Nikolic, A. Barney, and P. Garrard, "A feasibility study of speech recording using a contact microphone in patients with possible or probable alzheimer's disease to detect and quantify repetitions in a natural setting," Alzheimer's \& Dementia, vol. 8, no. 4, pp. P490-P491, 2012

[21] J. Wilson, A. Tingle, and H. Loveday, "Dehydration in elderly care home residents: a scoping review of associated morbidity and prevention strategies," International Journal of Evidence-Based Healthcare, vol. 11, no. 3, pp. 258-259, 2013.

[22] A. Carmichael, M. Rice, S. Lindsay, and P. Olivier, "itv as a platform for rich multimedia reminders for people with dementia," in Changing Television Environments. Springer, 2008, pp. 308-317. 